\title{
Single nucleotide polymorphisms in the intergenic region between metformin transporter OCT2 and OCT3 coding genes are associated with short-term response to metformin monotherapy in type 2 diabetes mellitus patients
}

\author{
Linda Zaharenko', Ineta Kalnina', Kristine Geldnere, 2,3, Ilze Konrade ${ }^{4,7}$, Solveiga Grinberga5, Jozef Židzik ${ }^{6}$, \\ Martin Javorský6, Aivars Lejnieks,7, Liene Nikitina-Zake1, Davids Fridmanis', Raitis Peculis', \\ Ilze Radovica-Spalvina1, Dace Hartmane ${ }^{5}$, Osvalds Pugovics $^{5}$, Ivan Tkáč ${ }^{6}$, Lucia Klimčáková6, Valdis Pīāgs 2,3,* \\ and Janis Klovins ${ }^{1, *}$
}

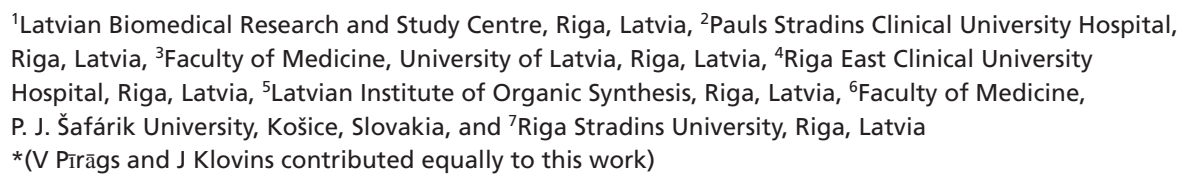

Correspondence should be addressed to J Klovins

Email

klovins@biomed.lu.lv

\begin{abstract}
Objective(s): High variability in clinical response to metformin is often observed in type 2 diabetes (T2D) patients, and it highlights the need for identification of genetic components affecting the efficiency of metformin therapy. Aim of this observational study is to evaluate the role of tagSNPs (tagging single nucleotide polymorphisms) from genomic regions coding for six metformin transporter genes with respect to the short-term efficiency.

Design: 102 tagSNPs in 6 genes coding for metformin transporters were genotyped in the group of 102 T2D patients treated with metformin for 3 months.

Methods: Most significant hits were analyzed in the group of 131 T2D patients from Slovakia. Pharmacokinetic study in 25 healthy nondiabetic volunteers was conducted to investigate the effects of identified polymorphisms.

Results: In the discovery group of 102 patients, minor alleles of rs3119309, rs7757336 and rs 2481030 were significantly nominally associated with metformin inefficiency $\left(P=1.9 \times 10^{-6}\right.$ to $\left.8.1 \times 10^{-6}\right)$. Effects of rs 2481030 and rs7757336 did not replicate in the group of 131 T2DM patients from Slovakia alone, whereas rs 7757336 was significantly associated with a reduced metformin response in combined group. In pharmacokinetic study, group of individuals harboring risk alleles of rs7757336 and rs 2481030 displayed significantly reduced $A U C_{\infty}$ of metformin in plasma.

Conclusions: For the first time, we have identified an association between the lack of metformin response and SNPs rs3119309 and rs7757336 located in the 5' flanking region of the genes coding for Organic cation transporter 2 and rs 2481030 located in the $5^{\prime}$ flanking region of Organic cation transporter 3 that was supported by the results of a pharmacokinetic study on 25 healthy volunteers.
\end{abstract}

European Journal of Endocrinology

(2016) 175, 531-540 


\section{Introduction}

Metformin (1,1-dimethylbiguanide) is prescribed to at least 120 million people worldwide to treat hyperglycemia (1). It is recommended as the first drug of choice for type 2 diabetes (T2D) according to the guidelines of the ADA (American Diabetes Association) and EASD (European Association for the Study of Diabetes), unless not tolerated or contraindicated $(2,3)$. Metformin provides approximately $1-1.5 \%$ improvement of $\mathrm{HbA}_{1 \mathrm{c}}$ and decreases insulin resistance $(4,5)$. A feature of the metformin therapy is the high variability in glycemic response ranging from good clinical response up to showing no benefit, and high prevalence of common side effects $(5-30 \%)(6,7,8)$. Up to about a third of the patients using metformin monotherapy were unable to achieve the $\mathrm{HbA}_{1 \mathrm{c}}$ target of $7 \%(53 \mathrm{mmol} / \mathrm{mol})$ as a treatment goal (9).

Metformin is not metabolized in the human body (10); its passive diffusion through the cell membranes is very limited (11). Its distribution, transportation to the target tissues and subsequent excretion from the organism are ensured by organic cation transporters (OCTs), multidrug and toxin extrusion antiporters (MATEs) and plasma membrane monoamine transporter (PMAT) to which metformin is a substrate. PMAT (SLC29A4) is expressed in human small intestine tissues and is thought to be involved in the gastrointestinal absorption of metformin (12). Organic cation transporter 1, OCT1 (SLC22A1), is expressed mainly in the liver and is a major mediator for the metformin uptake into the blood stream from enterocytes and the hepatic uptake of metformin (13). Organic cation transporter 3, OCT3 (SLC22A3), has a diverse expression pattern and is thought to mediate metformin uptake in the gastrointestinal tract, muscles, heart and liver; thus, it possibly may balance decreased function of OCT1 and PMAT $(13,14,15)$. Organic cation transporter 2, OCT2 (SLC22A2), is expressed mainly in the kidney, where it transports metformin into the proximal tubule cells (16). Finally, multidrug and toxin extrusion antiporters 1 and 2, MATE1 and MATE2K (SLC47A1, SLC47A2) are expressed in brush border of the renal proximal tubule and excrete metformin into the urine $(17,18)$.

Genome-wide complex trait analysis (GCTA) results showed that contribution of heritability in glycemic response to metformin could be $20-34 \%$ and suggested that mainly a spectrum of many genetic variants with small or moderate effect is involved (19). Results of the first genome-wide association study (GWAs) on the longterm efficiency of metformin support this hypothesis as the polymorphism rs11212617, located near the ataxiatelangiectasia-mutated gene (ATM) and significantly associated with metformin response, could explain only approximately $2.5 \%$ of its variability (20). Several case-control pharmacogenetic studies have investigated the associations between metformin efficiency and pharmacokinetic parameters with SNPs in OCT1, OCTN1, OCT2, OCT3, MATE1 and MATE2K coding genes $(21,22,23,24)$. Current findings indicate that research on metformin efficiency requires complex methods involving the identification of genetic variability in metformin transporters and pharmacokinetic parameters obtained in different mediums (like plasma, erythrocytes and urine) simultaneously.

In this study, we aimed to evaluate the clinical consequences of systematically selected tagSNPs from genomic regions coding for six metformin transporter genes with respect to the relatively short-term efficiency, where the metformin transporter effects may be the most prominent.

\section{Research design and methods}

\section{Study group}

Study was based on a prospective OPTIMED cohort that was launched in 2010 in the framework of the Latvian National Research Programme BIOMEDICINE. 28 endocrinologists from health care centers and hospitals were involved in the recruitment of patients with T2D to maintain up to a 3-year long follow-up study. All participants were included in the government-funded Genome Database of Latvian Population (LGDB) (27). Inclusion criteria were the following: patients with ICD-10 E11 diagnosis (fasting blood glucose test result $\geq 7 \mathrm{mmol} / \mathrm{L}$ and/or OGT test result $\geq 11 \mathrm{mmol} / \mathrm{L}$ ), drug naïve, over 18 years old, signed informed consent and lack of pregnancy in women. Baseline data on other diagnoses, history of gestational diabetes, anthropometric measurements (height, weight, waist circumference and blood pressure), intolerance of antidiabetic drugs and biochemical analysis were gathered. Overall, 313 prospective study participants $(\mathrm{OM})$ were recruited in the study on January 2015. 102 patients who have received metformin monotherapy for 3 months and had corresponding $\mathrm{HbA}_{1 \mathrm{c}}$ measurements $(95.5 \pm 9.0$ days after beginning of the therapy) were selected for this study. Time before baseline HbA1c measurement and start of the therapy was $<30$ days and was not significantly associated 
with $\triangle$ HbA1c values. OPTIMED project protocol was approved by the Central Medical Ethics Committee of Latvia (Protocol No. 01-29.1/22).

In Slovakia, the study was conducted in a university hospital setting. T2D was diagnosed in patients according to the criteria of the American Diabetes Association. The Louis Pasteur University Hospital Review Board gave ethical approval for this study. All participating subjects gave a written consent to be included in the study. 148 patients of Caucasian origin were recruited from three out-patient clinics. Patients with malignancies, another endocrine disorders, chronic kidney disease stage 3-5, severe liver disease and systemic inflammatory disease were excluded. Only drug-naïve patients with $\mathrm{HbA}_{1 \mathrm{c}}$ in the range of $6.5-11 \%(48-97 \mathrm{mmol} / \mathrm{mol})$ were included. Baseline $\mathrm{HbA}_{1 \mathrm{c}}$ measurement was performed within 1 week before the treatment initiation and second measurement was taken after 6 months of metformin monotherapy. 131 patients were further included in the study as they had $\mathrm{HbA}_{1 \mathrm{c}}$ measurements in the corresponding period (101.5 \pm 20.6 days).

Twenty-five volunteers from Latvia in the pharmacokinetic study group ranged between the ages of 22 and 37 years and have been evaluated to be healthy by a medical doctor. All participants have signed informed consent, and the study was approved by the Committee of Ethics (Nr.3000610-18L). Study group and determination of metformin in blood are described in Supplementary methods (see section on supplementary data given at the end of this article).

\section{SNP selection and genotyping}

We developed a genotyping panel using HaploView 4.2 (27 genome release) for SNP selection in CEU+TSI population. Due to the relatively small expected sample size, only variations exceeding minor allele frequency (MAF) of 0.05 in Caucasians were included. Altogether 192 SNPs and tagSNPs in regions covering the 52 genes that are previously reported as influencing the efficiency and tolerability of peroral antidiabetic therapy were selected. For a particular study, 108 tagSNPs located in OCT1 (SLC22A1), OCT2 (SLC22A2), OCT3 (SLC22A3), MATE1 (SLC47A1), MATE2K (SLC47A2) and PMAT (SLC29A4) regions were used. DNA samples for genotyping analysis were provided by LGDB. Genotyping was performed in all patients using GoldenGate Genotyping Assay with VeraCode technology (Illumina, San Diego, CA, USA) according to the manufacturer's instructions. One positive control sample in each assay plate was used; 19 duplicate samples were randomly distributed among the plates. Concordance rate of the randomly distributed duplicate samples was $99.7 \%$. The GeneCall threshold was set to 0.25 , and samples with call rates lower than 0.9 were excluded. The cluster images of signal intensity were reviewed manually for all SNPs. 18 from 192 SNPs were excluded from the panel after quality control due to failed genotyping or weak genotype separation due to low fluorescence. Primary data analyses were performed using Illumina GenomeStudio software as previously reported (26).

In the patient group from Slovakia, OCT2 rs7757336 and OCT2-OCT3 rs2481030 polymorphisms were analyzed by high-resolution melting analysis after realtime PCR in the presence of LCGreen Plus dye and an unlabeled probe on Eco Real-Time PCR System (Illumina). Genotypes were identified using Eco Software 4.1. Genotyping success rate for all the examined variants was $100 \%$, and duplicate genotyping concordance was $100 \%$ (15 samples for each SNP. Where available, 5 samples for each genotype were used).

Genetic testing of rs2481030 and rs7757336 in pharmacokinetic group was carried out using the Applied Biosystems TaqMan SNP (Applied Biosystems). Genotyping assay was performed with a modified protocol using $4.75 \mathrm{~mL}$ TaqMan Genotyping Mix, $0.25 \mathrm{~mL}$ SNP genotyping assay and $5 \mathrm{~mL}$ Millipore $\mathrm{H}_{2} \mathrm{O}$ on a 7500 Real-Time PCR system (Applied Biosystems). AutoCaller 1.1 (Applied Biosystems) software was used to assign genotype calls for all samples simultaneously (genotype call rate $-100 \%$, concordance rate $-100 \%$ ).

\section{Statistical analysis}

To assess the genotyping quality, statistical analysis was performed with the PLINK v1.07 software (http://pngu. mgh.harvard.edu/purcell/plink/) (27). Two SNPs from genotyping panel were excluded due to deviation from the Hardy-Weinberg equilibrium $P<0.05$ in the controls of discovery group. Altogether 102 tagSNPs with a genotyping rate of $97.2 \%$ in 102 individuals were used in further statistical analysis. Statistical power was calculated using Quanto software (Natara Software, Naperville, IL, USA). Our sample size provided $80 \%$ power (at $P=0.05$ ) to detect an odds ratios (ORs) from 1.55 to 2.9 depending on MAF of SNPs.

Tidwell-Box linearity test, standardized residual values, standard errors of mean (s.E.M.) of independent variables and Pearson's $r$ were obtained using SPSS 13.0 
(Standard version, Chicago, IL, USA) to analyze the quality of data and confirm the use of samples in logistic and linear regressions performed. The Durbin-Watson test, Kolmogorov-Smirnov test and Shapiro-Wilk test, VIF and tolerance and standardized residuals were obtained using SPSS 13.0 to analyze the quality of data retrieved from pharmacokinetic study.

Logistic regression of responders vs non-responders (reduction vs no reduction or increase in $\mathrm{HbA}_{1 \mathrm{c}}$ ) was performed using PLINK 1.07 open software assuming an additive mode of inheritance to estimate the association of SNPs with non-responsiveness after 3 months of metformin monotherapy in the discovery and replication groups and using a number of cofactors (age, sex, BMI, $\mathrm{HbA}_{1 \mathrm{c}}$, days of treatment, dose of metformin and creatinine clearance) to adjust the analysis for other non-genetic factors. Linear regression was performed using PLINK 1.07 open software to estimate the association of SNPs with absolute changes in the obtained pharmacokinetic parameters and to correct for covariates (creatinine clearance, age, sex and weight).

HaploReg2 was used for exploring annotations of the noncoding genome at variants on the haplotype blocks and LD information from the 1000 Genomes Project (http://www.broadinstitute.org/mammals/haploreg/ haploreg.php). IMPUTE version 2 was used for a genotype imputation and haplotype phasing with combined data from 1000 Genomes Pilot project and HapMap 3 (28) (https://mathgen.stats.ox.ac.uk/impute/ impute_v2.html). The 1000 Genomes project browser (http://browser.1000genomes.org/index.html), which contains the database of phase 3 autosomal variants, was used to predict the linkage disequilibrium among SNPs of interest.

\section{Results}

\section{Genetic association study in discovery and replication groups}

Characteristics of T2D patients included in the study are given in Table 1. Baseline $\mathrm{HbA}_{1 \mathrm{c}}$ level was similar in both groups; however, the discovery group showed significantly better treatment results in a shorter period in comparison with the replication group $\left(\Delta \mathrm{HbA}_{1 \mathrm{c}}\right.$, $0.9 \%$ vs $0.6 \%$ ). BMI and dose of metformin showed significant differences between both the groups. $\Delta \mathrm{HbA}_{1 \mathrm{c}}$ values were not normally distributed. Thus, Tidwell-Box linearity test ( $P$ value $>0.05$ ), Cook's distance values $<1$, standardized residual values $<2.58$ and under maximum leverage value confirmed the use of logistic regression to ascertain the effects of age, creatinine clearance, baseline $\mathrm{BMI}$ and $\mathrm{HbA}_{1 \mathrm{c}}$, sex, length of therapy, dose of metformin and 102 SNPs of the 6 metformin transporters (OCT1, OCT2, OCT3, MATE1, MATE2-K and PMAT) on the likelihood that participants have non-responder phenotype (no changes vs increased $\mathrm{HbA}_{1 \mathrm{c}}$ level) after 3 months of metformin monotherapy as described previously (29).

Data for the associations of all SNPs in the discovery group are shown in Supplementary Table 1. In total, 26 SNPs were nominally $(P<0.05)$ associated, whereas only 3 SNPs remained significantly associated after the correction for multiple testing. Table 2 shows 3 SNPs that were significantly associated with non-responder phenotype after Bonferroni correction, rs2481030, rs7757336 and rs3119309. The 1000 Genomes project browser (http://browser.1000genomes.org/index.html) predicted a high linkage disequilibrium $\left(\mathrm{D}^{\prime}=1.0\right)$ among rs7757336, rs3119309 and rs2481030 in the Caucasians

Table 1 Characteristics of T2D patient groups from Latvia and Slovakia.

\begin{tabular}{l} 
Characteristics \\
\hline Male, $n(\%)$ \\
Female, $n(\%)$ \\
Mean age \pm S.D., years \\
Mean $\mathrm{BMI} \pm$ S.D., $\mathrm{kg} / \mathrm{m}^{2}$, baseline \\
Creatinine clearance \pm S.D., $\mathrm{mL} / \mathrm{min}$ \\
Dose of metformin \pm s.D., $\mathrm{mg} /$ per day \\
Non-responders to the treatment (decrease of HbA1c, bilance) $n,(\%)$ \\
Days between $\mathrm{HbA} 1 \mathrm{c}$ measurments \pm S.D. \\
HbA1c \pm S.D., $\%, \mathrm{mmol} / \mathrm{mol}$, baseline \\
HbA1c \pm S.D., \%, $\mathrm{mmol} / \mathrm{mol}$, after treatment, \% \\
Decrease of HbA1c \pm S.D., after treatment, $\%$
\end{tabular}

\begin{tabular}{l}
\hline Discovery group $(n=102)$ \\
\hline $33(32.4)$ \\
$69(67.6)$ \\
$59.7 \pm 10.6$ \\
$33.8 \pm 4.8$ \\
$120.1 \pm 43.7$ \\
$1525.0 \pm 533.5$ \\
$18(17.6)$ \\
$95.5 \pm 9.0$ \\
$7.4 \pm 1.5(57 \pm 16.4)$ \\
$6.5 \pm 0.6(48 \pm 6.6)$ \\
$0.9 \pm 1.3$
\end{tabular}

\begin{tabular}{c}
\hline Replication group $(n=131)$ \\
\hline $\mathbf{6 7}(\mathbf{5 1 . 1})$ \\
$\mathbf{6 4}(\mathbf{4 8 . 9 )}$ \\
$57.4 \pm 10.7$ \\
$\mathbf{3 1 . 4} \pm \mathbf{4 . 5}$ \\
$105.4 \pm \mathbf{3 7 . 1}$ \\
$1053.1 \pm \mathbf{4 8 6 . 4}$ \\
$19(14.5)$ \\
$101.5 \pm 20.6$ \\
$7.6 \pm 1.0(60 \pm 10.9)$ \\
$7.0 \pm 0.7(53 \pm 7.7)$ \\
$\mathbf{0 . 6} \pm \mathbf{0 . 9}$
\end{tabular}

\begin{tabular}{c}
\hline $\boldsymbol{P}$ values \\
\hline $\mathbf{0 . 0 0 6}$ \\
$\mathbf{0 . 0 0 6}$ \\
0.104 \\
$<\mathbf{0 . 0 0 0 1}$ \\
$\mathbf{0 . 0 0 6}$ \\
$<\mathbf{0 . 0 0 0 1}$ \\
0.644 \\
$\mathbf{0 . 0 0 6}$ \\
0.225 \\
$<\mathbf{0 . 0 0 0 1}$ \\
$\mathbf{0 . 0 3 9}$
\end{tabular}

$P$ values $<0.05$ are marked with bold. $P$ values were assessed using $t$-test and comparison of proportions. S.D., standard deviation; BMI, body mass index. 
Table 2 SNPs associated with efficiency of metformin monotherapy in patients from Latvia and Slovakia.

\begin{tabular}{|c|c|c|c|}
\hline \multirow[b]{2}{*}{ SNP } & & \multicolumn{2}{|c|}{ Genotype number } \\
\hline & & Non-responders & Responders \\
\hline \multirow[t]{3}{*}{ rs7757336 } & Discovery group & $2 / 9 / 7$ & $0 / 14 / 70$ \\
\hline & Replication group & $0 / 7 / 12$ & $1 / 22 / 89$ \\
\hline & Combined analysis & $2 / 16 / 19$ & $1 / 36 / 159$ \\
\hline \multirow[t]{3}{*}{ rs3119309 } & Discovery group & $1 / 8 / 9$ & $0 / 9 / 75$ \\
\hline & Replication group & NA & NA \\
\hline & Combined analysis & NA & NA \\
\hline \multirow[t]{3}{*}{ rs2481030 } & Discovery group & $10 / 7 / 1$ & $8 / 38 / 38$ \\
\hline & Replication group & $2 / 9 / 8$ & $10 / 50 / 52$ \\
\hline & Combined analysis & $12 / 16 / 9$ & $18 / 88 / 90$ \\
\hline
\end{tabular}

\begin{tabular}{c}
\hline OR $(95 \% \mathrm{Cl})^{\dagger}$ \\
\hline $50.360(5.998-422.900)$ \\
$1.323(0.4089-4.282)$ \\
$4.029(1.956-8.301)$ \\
$26.580(4.631-152.500)$ \\
NA \\
NA \\
$13.700(3.435-54.670)$ \\
$1.279(0.563-2.907)$ \\
$2.933(1.674-5.137)$ \\
\hline
\end{tabular}

\begin{tabular}{|c|c|}
\hline$P^{\dagger}$ & $\boldsymbol{P}^{*}$ \\
\hline $3.06 * e^{-4}$ & 0.002 \\
\hline 0.640 & 0.826 \\
\hline $1.578 * e^{-4}$ & $3.137^{*} e^{-4}$ \\
\hline $2.34^{*} e^{-4}$ & 0.001 \\
\hline NA & NA \\
\hline NA & NA \\
\hline $2.09 * e^{-4}$ & 0.002 \\
\hline 0.557 & 0.342 \\
\hline $1.686^{*} e^{-4}$ & 0.052 \\
\hline
\end{tabular}

Discovery group, Latvia, $n=102$ patients, Replication group, Slovakia, $n=131{ }^{\dagger} \mathrm{OR}$ and $P$ values estimated from logistic regression adjusted for age, sex, baseline BMI, number of treatment days, dose of metformin $\mathrm{mg} /$ per day, creatinine clearance (Cockroft-Gault equation).

${ }^{\ddagger} P$ value estimated from logistic regression adjusted for age, sex, baseline BMl, dose of metformin mg/per day, creatinine clearance (Cockroft-Gault equation), number of treatment days and baseline $\mathrm{HbA}_{1 c} . P$ values significant after Bonferroni correction are marked bold.

(CEU) with variable $r^{2}$ due to differences between MAFs $\left(r^{2}=0.182-0.657\right)$.

The logistic regression model of combined minor alleles in the discovery group (count of minor alleles of rs2481030 and rs7757336) was statistically significant $\left(\chi^{2}(7)=67.729, P<0.001\right)$. The model explained $80.0 \%$ (Nagelkerke $R^{2}$ ) of the variance in response to metformin, and carriers of minor alleles were 12.8 times more likely to exhibit non-responder phenotypes than participants with reference alleles. Longer time of the therapy, higher baseline $\mathrm{HbA}_{1 \mathrm{c}}$ and women gender were associated with a lower likelihood of exhibiting non-responder phenotype $(P<0.05)$. Wide confidence intervals of models can be explained by small sample size and not as a consequence of the high standard errors as s.E.M. values for all logistic regression models $\leq 1.01$ and Pearson's $r<0.8$.

SNP imputation of OCT1/OCT2/OCT3 locus was performed, but none of the imputed SNPs displayed stronger association compared with rs2481030, rs7757336 and rs3119309.

Two SNPs, rs7757336 and rs2481030, were selected for replication from a group of 131 Slovakian T2D patients (replication group). In total, 148 Slovakian patients were included in a 6-month duration study. Of them, we had data on HbA1c from a 3-month visit in 131 patients, and these data were analyzed. Minor allele frequencies in replication group showed decreased MAF of SNPs rs7757336 $(0.118,0.132)$ and rs $2481030(0.317,0.397)$ in comparison with the discovery group and HapMap data (0.173, 0.398 respectively).

We found no significant associations of SNPs rs7757336 and rs2481030 (Omnibus test $P$ value $>0.05$ ) with non-responder phenotype in group from Slovakia after 3 months of metformin monotherapy in a model with covariates sex, age, BMI, length of therapy, dose (mg/per day), baseline $\mathrm{HbA}_{1 \mathrm{c}}$ and creatinine clearance.

On analysis of a combined group (233 participants), logistic regression model is statistically significant $\left(\chi^{2}(7)=76.070, P<0.001\right)$. The model included a number of minor alleles of rs2481030 or rs7757336 and age, sex, baseline $\mathrm{BMI}$ and $\mathrm{HbA}_{1 \mathrm{c}}$, number of treatment days, dose of metformin $\mathrm{mg} /$ per day and creatinine clearance as covariates. Carriers of minor alleles were 2.00 times more likely to exhibit non-responder phenotypes than participants with reference alleles $(P=0.002)$. Higher creatinine clearance, higher baseline $\mathrm{HbA}_{1 \mathrm{c}}$, age and women gender were associated with a lower likelihood of exhibiting non-responder phenotype $(P<0.05)$.

\section{Pharmacokinetic study}

Association of rs2481030 and rs7757336 with metformin pharmacokinetics was analyzed in a group of 25 participants. Phenotypic and biochemical data of those subjects are displayed in Table 3. Nine heterozygotes (AG) and 4 homozygotes (GG) of rs2481030 risk allele were identified. Eight heterozygotes (AC) of rs7757336 risk allele were identified, all being carriers of minor alleles of rs2481030 (AG and GG genotypes). Due to the small group size, all risk alleles were counted for each participant and used as covariates for linear regression analysis. For clarity of presentation, 12 participants with 0 risk alleles were assigned to the 'reference' group, whereas 13 participants with at least 1 risk allele were assigned to the 'risk group'. There are no significant differences in the sex, age, weight and creatinine clearance between the groups. 
Table 3 Characteristics and pharmacokinetics parameters of 25 healthy participants in relation to combined alleles of rs 2481030 and rs7757336 associated with metformin inefficiency after a single-dose oral administration of $500 \mathrm{mg}$ metformin. Data are shown as mean value \pm S.D.

\begin{tabular}{|c|c|c|c|c|}
\hline \multirow[b]{2}{*}{ Characteristics } & \multirow[b]{2}{*}{ Study sample } & \multicolumn{3}{|c|}{ Comparison of groups } \\
\hline & & Reference $(n=12)$ & Risk group $(n=13)$ & $P$ values \\
\hline Male $n, \%$ & $9(36.00)$ & $3(25.00)$ & $6(46.15)$ & $0.473^{\ddagger}$ \\
\hline Weight, kg, S.D. & $72.68 \pm 13.32$ & $71.58 \pm 16.59$ & $73.69 \pm 10.01$ & $0.701^{\ddagger}$ \\
\hline Age, years, S.D. & $26.44 \pm 3.99$ & $26.17 \pm 4.73$ & $26.69 \pm 3.35$ & $0.752^{\ddagger}$ \\
\hline Creatinine clearance, $\mathrm{mL} / \mathrm{min} / \mathrm{BSA}, \pm$ S.D. & $121.06 \pm 13.72$ & $118.54 \pm 16.34$ & $123.38 \pm 10.93$ & $0.390^{\ddagger}$ \\
\hline $\mathrm{AUC}_{\infty}, \mu \mathrm{g} * \mathrm{~h} / \mathrm{mL}, \pm \mathrm{S} . \mathrm{D}$ & $5.42 \pm 1.62$ & $6.30 \pm 1.51$ & $4.62 \pm 1.29$ & 0.009 \\
\hline$C_{\max }, \mu g^{*} \mathrm{~mL}$, plasma, \pm S.D. & $0.72 \pm 0.24$ & $0.84 \pm 0.25$ & $0.60 \pm 0.18$ & 0.022 \\
\hline$T_{\max }$, plasma, $h, \pm$ S.D. & $2.32 \pm 1.25$ & $2.42 \pm 1.51$ & $2.23 \pm 1.01$ & NS \\
\hline$C_{\max } \mu \mathrm{g} / \mathrm{mL}$, erythrocytes, \pm S.D. & $0.15 \pm 0.06$ & $0.16 \pm 0.07$ & $0.14 \pm 0.05$ & NS \\
\hline Estimated half-life, h, \pm S.D. & $4.44 \pm 0.62$ & $4.41 \pm 0.56$ & $4.46 \pm 0.70$ & NS \\
\hline CL/F, L/h, \pm S.D. & $51.40 \pm 16.79$ & $42.47 \pm 9.50$ & $59.64 \pm 18.11$ & 0.010 \\
\hline V/F, L, \pm S.D. & $333.56 \pm 146.18$ & $268.04 \pm 59.66$ & $394.03 \pm 176.70$ & NS \\
\hline Metformin excreted in the urine, $\%$ of dose \pm S.D. & $39.09 \pm 12.94$ & $42.19 \pm 16.42$ & $36.23 \pm 8.31$ & NS \\
\hline
\end{tabular}

${ }^{\ddagger} P$ value derived from $t$-test. All other $P$ values derived from linear regression with covariates: number of negative alleles, age, sex, weight, creatinine clearance (corrected for body surface area (BSA)). NS: linear regression model is not significant (Omnibus test).

The concentration of metformin in plasma was higher in the individuals from the reference group compared with those in the risk group in 4 sampling points (Fig. 1).

Quality measures for the regression analysis including combined number of risk alleles of rs7757336 and rs2481030 polymorphisms and covariates - weight, age, sex and creatinine clearance (calculated from 24-h urine samples and corrected for body surface area) - were as follows: $R^{2}=44.2 \%$, result of the Durbin-Watson test $=1.83$, VIF was $1.06-1.56$ and tolerance $0.64-0.95$ and standardized residuals of linear regression with values $< \pm 2.17$ were normally distributed $(P=0.2)$ (Table 3).

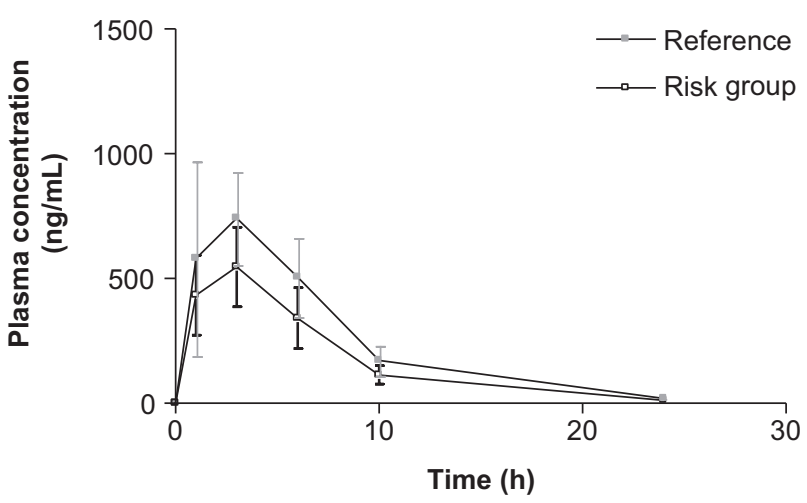

Figure 1

Concentration of metformin in plasma of 25 healthy individuals grouped based on the presence of the risk alleles after a single-dose oral administration of $500 \mathrm{mg}$ metformin.
AUC $_{\infty}$ of metformin plasma was significantly lower $(P=0.009)$ in the risk group $(4.62 \pm 1.29 \mu \mathrm{g} / \mathrm{h} / \mathrm{mL})$ vs reference group $(6.30 \pm 1.51 \mu \mathrm{g} / \mathrm{h} / \mathrm{mL})$ in linear regression analysis. Model including number of risk alleles, age, sex, weight and creatinine clearance statistically significantly predicted metformin $\mathrm{AUC}_{\infty}$ in plasma (sig. $F$ change $(5,19)=3.005, P=0.036)$. Weight $(P=0.037)$ and number of minor alleles $(P=0.009)$, but not age $(P=0.775)$, sex $(P=0.581)$ and creatinine clearance $(P=0.728)$ were statistically significant variables to the prediction. $C_{\max }$ in plasma was significantly increased in reference group $(0.84 \pm 0.25$ vs $0.60 \pm 0.18 \mu \mathrm{g} / \mathrm{mL}, P=0.022)$, and apparent clearance $(\mathrm{CL} / \mathrm{F})$ was significantly higher in risk group $(59.64 \pm 18.11 \mathrm{~L} / \mathrm{h}$ vs $42.47 \pm 9.50 \mathrm{~L} / \mathrm{h}, P=0.01)$. There were no significant differences between groups with respect to $\mathrm{T}_{\max }$ in plasma, $\mathrm{C}_{\max }$ in erythrocytes, estimated half-life and apparent volume of distribution, as well as dose of metformin excreted in the urine $24 \mathrm{~h}$ after drug administration. $T_{\max }$ in erythrocytes was not analyzed due to low variability in study sample.

\section{Discussion}

In this study, we show that minor alleles of SNPs rs3119309, rs7757336 and rs2481030 located in the intergenic region between OCT2 and OCT3 coding genes (SLC22A2 and SLC22A3) are significantly associated with metformin inefficiency in the 233 newly diagnosed and well-described T2D patients. Testing of pharmacokinetic effects of these genetic markers was performed in 25 nondiabetic volunteers from Latvia. 
Logistic analysis was performed using robust segregation of outcomes in the responder group showing decrease in $\mathrm{HbA}_{1 \mathrm{c}}$ vs non-responder group displaying no change or even increasing levels of $\mathrm{HbA}_{1 \mathrm{c}} 3$ months after the beginning of metformin monotherapy. The main reason to choose this type of outcome is due to our intention to perform robust analysis that is better suited for the investigation in the small, well-phenotyped group (29). A number of studies investigating the efficiency of metformin including the only GWAS performed so far (20) have used the $\Delta \mathrm{HbA}_{1 \mathrm{c}}$ or achievement of treatment goal of $<6.5$ or $7 \% \mathrm{HbA}_{1 \mathrm{c}}(48-53 \mathrm{mmol} / \mathrm{mol})$ as an outcome. However, patients with high baseline $\mathrm{HbA}_{1 \mathrm{c}}$ level will usually have larger $\Delta \mathrm{HbA}_{1 \mathrm{c}}$ in comparison with other participants due to the natural limits. Nevertheless, they often will be included in the non-response group if treatment goals are set as criteria even if absolute decrease of $\mathrm{HbA}_{1 \mathrm{c}}$ is substantial. This may explain the significant deviation of $\Delta \mathrm{HbA}_{1 \mathrm{c}}$ from the normal distribution in our research group. For the same reason, use of treatment goals can be misleading in the patients with extreme baseline $\mathrm{HbA}_{1 \mathrm{c}}$ values. Patients with high baseline $\mathrm{HbA}_{1 \mathrm{c}}$ will have larger $\mathrm{HbA}_{1 \mathrm{c}}$ decrease than patients with $\mathrm{HbA}_{1 \mathrm{c}}$ close to normal levels but more often will be classified as non-responders $(30,31)$.

As previously mentioned, GWAS identified an association with rs11212617 near ATM, whereas no association was found in any loci of known metformin transporter genes (20). Our results showing the association of SNPs in metformin transporter genes in contrast to this GWAS may be explained by relatively short time period used to estimate the efficiency of metformin in our study. Factors influencing the pharmacokinetics (e.g. organic cation transporters) will show their effects on efficiency mainly at the beginning of the treatment, especially in the case of metformin where gradual accumulation of drug has been observed in the organism over the time of treatment (21). Short time period is also less dependent on factors like diet, physical activities and adherence to the drug in comparison with the longterm treatment when metformin secondary failure due to decreasing body functions interferes. In addition, one should take into account the fact that initial metformin therapy is subject to change after the first 3 months of the therapy in cases where it failed to display the decrease of $\mathrm{HbA}_{1 \mathrm{c}}$. A study investigating metformin therapy outcomes assessed initial nonadherence to be $17 \%$ in participants starting metformin therapy. Onethird attempted another pharmacotherapy within 6 months, and side effects were found to be one of the major limiting factors $(32,33,34)$. Thus, the patients whose treatment inefficiency is caused by the defect in metformin transport may become eliminated from the studies involving longer observation times.

Our failure to replicate the association of identified SNPs in the replication group of 131 T2D participants from Slovakia may be explained by the differences in the phenotypical and genotypical characteristics of the groups. Among the phenotypical differences, significantly lower BMI, lower dose of metformin used and subsequently a lower reduction in $\mathrm{HbA}_{1 \mathrm{c}}$ were observed in the replication group in comparison with those in the discovery group. SNPs rs7757336 and rs2481030, age, sex, BMI, $\mathrm{HbA}_{1 \mathrm{c}}$, metformin dose and creatinine clearance were not associated with non-responder phenotype, indicating a strong influence of other genetic or environmental factors like diet, physical activities and adherence to the metformin. MAF of the investigated SNPs in the replication group was lower compared with that found in the discovery group, and linkage disequilibrium differed ( $r^{2}=0.255$ vs 0.194$)$ indicating significant genetic differences between populations. However, it should be noted that the effect direction in the Slovakian group was similar to that observed in the Latvian group (OR 1.32, although non-significant). The results from combined analysis probably correspond better with the real-life effect size of the polymorphism with response to metformin, which could have been inflated in the original Latvian study as a result of statistical phenomenon called the 'winner's curse'. The combined analysis was made across the wider spectrum of metformin dosage, which was also taken into account as one of the covariates.

In line with the results of the pharmacogenetic study, in the pharmacokinetic study, the minor alleles of rs775336 and rs2481030 were also consistently associated with lower concentration of metformin and $\mathrm{AUC}_{\infty}$ of the plasma in a small independent group of 25 healthy nondiabetic volunteers. Due to the limited number of participants, it is necessary to confirm these findings in studies with a larger number of subjects. However, if these findings hold true, this will support the influence of OCTs in pharmacokinetics of metformin and importance of short-term study design in cases where factors influencing bioavailability are investigated. No significant differences were found in observed $C_{\max }$ and $\mathrm{T}_{\max }$ in erythrocytes between controls and carriers of risk alleles indicating that observed changes of metformin pharmacokinetics cannot be explained by exposure time to metformin. When other pharmacokinetic measures were analyzed, we found that oral clearance $(\mathrm{CL} / \mathrm{F})$ is 
higher in the subjects with at least one inefficient allele, whereas apparent volume of distribution (V/F), half-life and total dose of metformin excreted in the first $24 \mathrm{~h}$ after administration were not different. It should be noted however that calculation of renal clearance and apparent volume of distribution were done assuming that there is no difference in the bioavailability of $500 \mathrm{mg}$ of metformin taken orally. This assumption may not be true as a number of factors including the genetic variation in transporter genes may alter the bioavailability. The calculated $\mathrm{CL} / \mathrm{F}$ and $\mathrm{V} / \mathrm{F}$ values are strongly related to $\mathrm{AUC}_{\infty}$ measurement, and their difference among groups should be interpreted with caution.

Variation in the bioavailability and volume of distribution is considered a major source of variation in the pharmacokinetics of metformin as it does not undergo metabolization in the human body and slow absorption is thought to be the rate-limiting factor in metformin disposition (10). It is not clear what proportion of metformin is sequestered in the enterocytes due to the effects of inward net flow and OCT related mechanism vs its paracellular route in human small intestine. If latter is true, the uptake and excretion remain the major factors influencing metformin plasma levels. Lower metformin level in plasma may indicate lower metformin uptake in the gastrointestinal tract by altered OCT3 transporter or can be explained by OCT2 variant that eliminates the drug more quickly.

Polymorphisms investigated in this study are noncoding and could be in linkage disequilibrium with causal SNPs within coding/regulatory regions of SLC22A2, $S L C 22 A 3$ or even $S L C 22 A 1$ resulting in altered transport activity or expression level in target tissues. In large GWAS, minor allele A of SNP rs3127573 (MAF 0.13) located near SLC22A2 $\left(r^{2}=0.96\right.$ with SNP rs3119309 investigated in our study) was identified to be associated with higher serum creatinine and lower estimated GFR (35). However, it is not clear how variants in OCT2 or its regulatory regions could be a basis for increased creatinine levels and decreased metformin levels simultaneously.

On the other hand, these can be explained by altered transport activity of creatinine and metformin transporter OCT3 (SLC22A3), which is expressed in the liver, small intestine and muscle tissue. Indeed, in the experiments with OCT2 and OCT3 expressing HEK293 cells (36), increased creatinine uptake in comparison with cells transfected with the null vector was identified, whereas such uptake was not observed in case of OCT1. Polymorphisms rs2292334, rs2048327, rs1810126 and rs3088442 were associated with reduced OCT3 mRNA levels, whereas a common variant of rs555754 was associated with a greater transcription rate and higher expression levels of OCT3 in the liver (37). While none of the above-mentioned SNPs altering the OCT3 transporter efficiency were in strong linkage disequilibrium with SNPs investigated in our study, rs2481030 and rs2481031 (LD with $r^{2}=0.99$ ) (http://www.broadinstitute. org/mammals/haploreg/haploreg_v2.php). Possibly, rs2481030 could be a marker of reduced uptake OCT3 variant or lower transporter expression level and subsequently characterized by slower metformin absorption from gastrointestinal tract and decreased uptake in the liver, muscle, heart and adipose tissues.

Major limitations of our study are the relatively small sample size, possible effects of co-medications and adherence. Variable co-medications in antidiabetic therapy are very common, however, the stratification based on this variable or analysis of patients without concomitant diseases would result in significant selection bias and insignificant sample size. Particular functional variants, for example, OCT1 risk alleles of metformin intolerance were not included in this study (38) mainly because of low allele frequency that would result in very low statistical power due to small sample size. Our findings should be evaluated in larger groups of participants. Further investigation should be conducted to confirm whether rs7757336, rs3119309 and rs2481030 are valid markers of metformin inefficiency and if they can be useful in the prediction of treatment response to metformin in the T2D patients.

\section{Supplementary data}

This is linked to the online version of the paper at http://dx.doi.org/10.1530/ EJE-16-0347.

Declaration of interest

The authors declare that there is no conflict of interest that could be perceived as prejudicing the impartiality of the research reported.

\section{Funding}

This study was supported by State Research Programme 'Development of advanced prevention strategies, treatment, diagnostic tools and methods, biomedical technologies for improving public health' and the National Research Programme 'Biomedicine for Public Health' (BIOMEDICINE) and European Social Fund within the project 'Support for Doctoral Studies at University of Latvia'.

\footnotetext{
Acknowledgements

The authors acknowledge Dr med. Ārija Fjodorova, Dr med. Inese Care, Dr med. Tatjana Kuplā, Dr med. Inguta Stūra, Dr med. Natālija Fokina, Dr med. Pēteris Voicehovičs, Dr med. Gita Erta, Dr med. Spodrite Keisa, Dr med. Valentīna Purmale, Dr med. Ilze Lagzdina, Dr med. leva Lase, Dr med. Nataḷja Šidlovska, Dr med. Una Gailiša, Dr med. Indra Štelmane,
} 
Dr med. Santa Broka, Dr med. Elīna Menise, Dr med. Larisa Kokare, Dr med. Simona Saukuma and Mārite Cirse for patient recruitment.

The authors acknowledge Genome Database of Latvian Population for providing data and DNA samples.

\section{References}

1 Viollet B, Guigas B, Sanz Garcia N, Leclerc J, Foretz M \& Andreelli F. Cellular and molecular mechanisms of metformin: an overview. Clinical Science 2012122 253-270. (doi:10.1042/CS20110386)

2 ADA. Diagnosis and classification of diabetes mellitus. Diabetes Care 201437 (Supplement 1) S81-S90. (doi:10.2337/dc14-S081)

3 Nathan DM, Buse JB, Davidson MB, Ferrannini E, Holman RR, Sherwin R \& Zinman B. Management of hyperglycemia in type 2 diabetes: a consensus algorithm for the initiation and adjustment of therapy: update regarding thiazolidinediones: a consensus statement from the American Diabetes Association and the European Association for the Study of Diabetes. Diabetes Care 200831 173-175. (doi:10.2337/dc08-9016)

4 Herman SL. Impaired glucose tolerance and metformin: clinical and mechanistic aspects. British Journal of Diabetes \& Vascular Disease 2002 2 177-183. (doi:10.1177/14746514020020030501)

5 Ou HY, Cheng JT, Yu EH \& Wu TJ. Metformin increases insulin sensitivity and plasma beta-endorphin in human subjects. Hormone and Metabolic Research 200638 106-111. (doi:10.1055/s-2006-925128)

6 Donnelly LA, Morris AD \& Pearson ER. Adherence in patients transferred from immediate release metformin to a sustained release formulation: a population-based study. Diabetes, Obesity \& Metabolism 200911 338-342. (doi:10.1111/j.1463-1326.2008.00973.x)

7 Brown JB, Conner C \& Nichols GA. Secondary failure of metformin monotherapy in clinical practice. Diabetes Care 201033 501-506. (doi:10.2337/dc09-1749)

8 Graham GG, Punt J, Arora M, Day RO, Doogue MP, Duong JK, Furlong TJ, Greenfield JR, Greenup LC, Kirkpatrick CM et al. Clinical pharmacokinetics of metformin. Clinical Pharmacokinetics 201150 81-98. (doi:10.2165/11534750-000000000-00000)

9 Esposito K, Chiodini P, Bellastella G, Maiorino MI \& Giugliano D. Proportion of patients at HbA1c target $<7 \%$ with eight classes of antidiabetic drugs in type 2 diabetes: systematic review of 218 randomized controlled trials with 78945 patients. Diabetes, Obesity \& Metabolism 201214 228-233. (doi:10.1111/j.1463-1326.2011.01512.x)

10 Pentikainen PJ, Neuvonen PJ \& Penttila A. Pharmacokinetics of metformin after intravenous and oral administration to man. European Journal of Clinical Pharmacology 197916 195-202. (doi:10.1007/BF00562061)

11 Proctor WR, Bourdet DL \& Thakker DR. Mechanisms underlying saturable intestinal absorption of metformin. Drug Metabolism and Disposition 200836 1650-1658. (doi:10.1124/dmd.107.020180)

12 Zhou M, Xia L \& Wang J. Metformin transport by a newly cloned proton-stimulated organic cation transporter (plasma membrane monoamine transporter) expressed in human intestine. Drug Metabolism and Disposition 200735 1956-1962. (doi:10.1124/ dmd.107.015495)

13 Nies AT, Koepsell H, Winter S, Burk O, Klein K, Kerb R, Zanger UM, Keppler D, Schwab M \& Schaeffeler E. Expression of organic cation transporters OCT1 (SLC22A1) and OCT3 (SLC22A3) is affected by genetic factors and cholestasis in human liver. Hepatology 200950 1227-1240. (doi:10.1002/hep.23103)

14 Muller J, Lips KS, Metzner L, Neubert RH, Koepsell H \& Brandsch M. Drug specificity and intestinal membrane localization of human organic cation transporters (OCT). Biochemical Pharmacology 200570 1851-1860. (doi:10.1016/j.bcp.2005.09.011)

15 Verhaagh S, Schweifer N, Barlow DP \& Zwart R. Cloning of the mouse and human solute carrier 22a3 (Slc22a3/SLC22A3) identifies a conserved cluster of three organic cation transporters on mouse chromosome 17 and human 6q26-q27. Genomics 199955 209-218. (doi:10.1006/geno.1998.5639)

16 Gründemann D, Köster S, Kiefer N, Breidert T, Engelhardt M, Spitzenberger F, Obermüller N \& Schömig E. Transport of monoamine transmitters by the organic cation transporter type 2, OCT2. Journal of Biological Chemistry 1998273 30915-30920. (doi:10.1074/ jbc.273.47.30915)

17 Otsuka M, Matsumoto T, Morimoto R, Arioka S, Omote H \& Moriyama Y. A human transporter protein that mediates the final excretion step for toxic organic cations. PNAS $2005 \mathbf{1 0 2}$ 17923-17928. (doi:10.1073/pnas.0506483102)

18 Tanihara Y, Masuda S, Sato T, Katsura T, Ogawa O \& Inui K. Substrate specificity of MATE1 and MATE2-K, human multidrug and toxin extrusions/H(+)-organic cation antiporters. Biochemical Pharmacology 200774 359-371. (doi:10.1016/j.bcp.2007.04.010)

19 Zhou K, Donnelly L, Yang J, Li M, Deshmukh H, Van Zuydam N, Ahlqvist E, Wellcome Trust Case Control Consortium, Spencer CC \& Groop L. Heritability of variation in glycaemic response to metformin: a genome-wide complex trait analysis. Lancet Diabetes \& Endocrinology 20142 481-487. (doi:10.1016/S2213-8587(14)70050-6)

20 GoDARTS and UKPDS Diabetes Pharmacogenetics Study Group, Wellcome Trust Case Control Consortium, Zhou K, Bellenguez C, Spencer CC, Bennett AJ, Coleman RL, Tavendale R, Hawley SA, Donnelly LA et al. Common variants near ATM are associated with glycemic response to metformin in type 2 diabetes. Nature Genetics 201143 117-120. (doi:10.1038/ng.735)

21 Christensen MM, Brasch-Andersen C, Green H, Nielsen F, Damkier P, Beck-Nielsen $\mathrm{H} \&$ Brosen $\mathrm{K}$. The pharmacogenetics of metformin and its impact on plasma metformin steady-state levels and glycosylated hemoglobin A1c. Pharmacogenetics and Genomics 201121 837-850. (doi:10.1097/FPC.0b013e32834c0010)

22 Tzvetkov MV, Vormfelde SV, Balen D, Meineke I, Schmidt T, Sehrt D, Sabolić I, Koepsell H \& Brockmöller J. The effects of genetic polymorphisms in the organic cation transporters OCT1, OCT2, and OCT3 on the renal clearance of metformin. Clinical Pharmacology \& Therapeutics 200986 299-306. (doi:10.1038/clpt.2009.92)

23 Tkáč I, Klimčáková L, Javorský M, Fabianová M, Schroner Z, Hermanová H, Babjaková E \& Tkáčová R. Pharmacogenomic association between a variant in SLC47A1 gene and therapeutic response to metformin in type 2 diabetes. Diabetes, Obesity \& Metabolism 201315 189-191. (doi:10.1111/j.1463-1326.2012.01691.x)

24 Becker ML, Visser LE, van Schaik RH, Hofman A, Uitterlinden AG \& Stricker BH. Interaction between polymorphisms in the OCT1 and MATE1 transporter and metformin response. Pharmacogenetics and Genomics 201020 38-44. (doi:10.1097/FPC.0b013e328333bb11)

25 Ciganoka D, Balcere I, Kapa I, Peculis R, Valtere A, NikitinaZake L, Lase I, Schiöth HB, Pirags V \& Klovins J. Identification of somatostatin receptor type 5 gene polymorphisms associated with acromegaly. European Journal of Endocrinology 2011165 517-525. (doi:10.1530/EJE-11-0416)

26 Radovica I, Fridmanis D, Vaivade I, Nikitina-Zake L \& Klovins J. The association of common SNPs and haplotypes in CETP gene with HDL cholesterol levels in Latvian population. PLOS ONE 20138 e64191. (doi:10.1371/journal.pone.0064191)

27 Purcell S, Neale B, Todd-Brown K, Thomas L, Ferreira MA, Bender D, Maller J, Sklar P, de Bakker PI, Daly MJ et al. PLINK: a tool set for whole-genome association and population-based linkage analyses. American Journal of Human Genetics 200781 559-575. (doi:10.1086/519795)

28 Howie BN, Donnelly P \& Marchini J. A flexible and accurate genotype imputation method for the next generation of genome-wide association studies. PLoS Genetics 20095 e1000529. (doi:10.1371/ journal.pgen.1000529)

29 Shinji Ichimori, Seiya Shimoda, Rieko Goto, Yasuto Matsuo, Takako Maeda, Noboru Furukawa, Junji Kawashima, Shoko Kodama, Taiji Sekigami, Satoshi Isami et al. Ezetimibe improves glucose 
metabolism by ameliorating hepatic function in Japanese patients with type 2 diabetes. Journal of Diabetes Investigation 20123 179-184. (doi:10.1111/j.2040-1124.2011.00147.x)

30 Sherifali D, Nerenberg K, Pullenayegum E, Cheng JE \& Gerstein HC. The effect of oral antidiabetic agents on A1C levels: a systematic review and meta-analysis. Diabetes Care 201033 1859-1864. (doi:10.2337/dc09-1727)

31 Bloomgarden ZT, Dodis R, Viscoli CM, Holmboe ES \& Inzucchi SE. Lower baseline glycemia reduces apparent oral agent glucose-lowering efficacy: a meta-regression analysis. Diabetes Care 200629 2137-2139. (doi:10.2337/dc06-1120)

32 Hermans MP, Delibasi T, Farmer I, Lohm L, Maheux P, Piatti P, Malvolti E, Jörgens S \& Charbonnel B. Effects of saxagliptin added to sub-maximal doses of metformin compared with uptitration of metformin in type 2 diabetes: the PROMPT study. Current Medical Research and Opinion 201228 1635-1645. (doi:10.1185/03007995.201 2.735646)

33 Florez H, Luo J, Castillo-Florez S, Mitsi G, Hanna J, Tamariz L, Palacio A, Nagendran S \& Hagan M. Impact of metformin-induced gastrointestinal symptoms on quality of life and adherence in patients with type 2 diabetes. Postgraduate Medicine 2010122 112-120. (doi:10.3810/pgm.2010.03.2128)
34 Nichols GA, Conner C \& Brown JB. Initial nonadherence, primary failure and therapeutic success of metformin monotherapy in clinical practice. Current Medical Research and Opinion 201026 2127-2135. (doi:10.1185/03007995.2010.504396)

35 Chambers JC, Zhang W, Lord GM, van der Harst P, Lawlor DA, Sehmi JS, Gale DP, Wass MN, Ahmadi KR, Bakker SJ et al. Genetic loci influencing kidney function and chronic kidney disease. Nature Genetics 201042 373-375. (doi:10.1038/ng.566)

36 Ciarimboli G, Lancaster CS, Schlatter E, Franke RM, Sprowl JA, Pavenstädt H, Massmann V, Guckel D, Mathijssen RH, Yang W et al. Proximal tubular secretion of creatinine by organic cation transporter OCT2 in cancer patients. Clinical Cancer Research 201218 1101-1108. (doi:10.1158/1078-0432.CCR-11-2503)

37 Chen L, Hong C, Chen EC, Yee SW, Xu L, Almof EU, Wen C, Fujii K, Johns SJ, Stryke D et al. Genetic and epigenetic regulation of the organic cation transporter 3, SLC22A3. Pharmacogenomics Journal 2013 13 110-120. (doi:10.1038/tpj.2011.60)

38 Mahrooz A, Parsanasab H, Hashemi-Soteh MB, Kashi Z, Bahar A, Alizadeh A \& Mozayeni M. The role of clinical response to metformin in patients newly diagnosed with type 2 diabetes: a monotherapy study. Clinical and Experimental Medicine 201515 159-65. (doi:10.1007/s10238-014-0283-8)

Received 18 April 2016

Revised version received 31 August 2016

Accepted 8 September 2016 